\title{
A reconfigurable layout for a self-structuring life- jacket-integrated antenna of a SAR system
}

\author{
Andrea Baroni and Paolo Nepa \\ Department of Information Engineering \\ University of Pisa, \\ Pisa, Italy \\ andrea.baroni@for.unipi.it,p.nepa@iet.unipi.it
}

\author{
Hendrik Rogier \\ Department of Information Technology \\ Ghent University \\ Ghent, Belgium \\ hendrik.rogier@intec.ugent.be
}

\begin{abstract}
A reconfigurable layout to implement a selfstructuring wearable antenna for the local user terminal of a Search and Rescue system (Cospas-Sarsat at $406 \mathrm{MHz}$ ) is presented. The proposed antenna layout has been designed for integration on a commercial life jacket for use in a maritime environment. The shape of the antenna can be reconfigured by means of RF switches to ensure the best possible transmission conditions, in terms of overall antenna efficiency, even if part of the antenna goes under the sea level. The shape of the components of the self-structuring antenna has been optimized for different antenna positions with respect to the water level and immersion angles.
\end{abstract}

\section{INTRODUCTION}

In recent years, a number of wearable antennas have been developed for the user terminals of SAR (Search and Rescue) applications in the Cospas-Sarsat band at $406 \mathrm{MHz}$ [1]-[2], where the radiating elements are integrated into the floating part of a life jacket. In this scenario, the antenna performance (i.e. reflection coefficient and radiation pattern) is affected by the presence of the wearer human body, the water vicinity, the antenna-body coupling variations related to the natural wearer movements. As a shipwreck randomly changes position, part of the antenna could be partially immersed under the water level and a high return loss would make the system useless for transmission by an emergency beacon (due to decreased gain) [3]. Furthermore, part of the antenna could also be damaged during the frenetic wearing operations.

In such a scenario, it is essential to minimize the sensitivity of the antenna impedance to human movements and environmental changes. A self-structuring antenna [4] could be a valuable solution for a Cospas-Sarsat user terminal antenna at $406 \mathrm{MHz}$. A self-structuring antenna is made of a combination of wires or strips interconnected by controllable switches. This setup enables dynamic changes on the radiating shape to ensure the best of all possible transmission conditions (in terms of overall antenna efficiency), even if part of the antenna goes under the sea level. The selection of a particular radiating shape is based on real-time reflection-coefficient monitoring through a proper algorithm implemented on a microcontroller. When the number of possible antenna shapes is large, optimization algorithms (such as a Genetic algorithm, an Ant Colony optimization algorithm or Simulated Annealing) can be used for the switching policy [5]. Self-structuring antennas have been proposed in the UHF band for FM signal reception [6] and television reception [7].

In this paper, a printed low-profile reconfigurable layout for a self-structuring antenna is proposed to operate with a SAR terminal at $406 \mathrm{MHz}$. The suggested solution has been optimized for being integrated on a commercial life-jacket. The antenna has been simulated using the commercial CST Microwave Studio ${ }^{\circledR}$ full-wave solver.

\section{ANTENNA DESIGN AND SIMULATION RESULTS}

The topology of the proposed antenna is depicted in Fig. 1a. The input port of the antenna is located at the center of a commercial life jacket floating element $(8 \mathrm{~cm}$ thick). Starting from the central point, the antenna follows a multi-branch meandered dipole design. The main branches are the inner branch (Fig. 1b) and the outer branch (Fig. 1c). Each copper strip is connected to the adjacent one by means of RF switches. At the end of every branch, some conducting elements could be added for fine tuning by applying single-pole single-throw switches. At all other locations, single-pole double-throw switches are used.

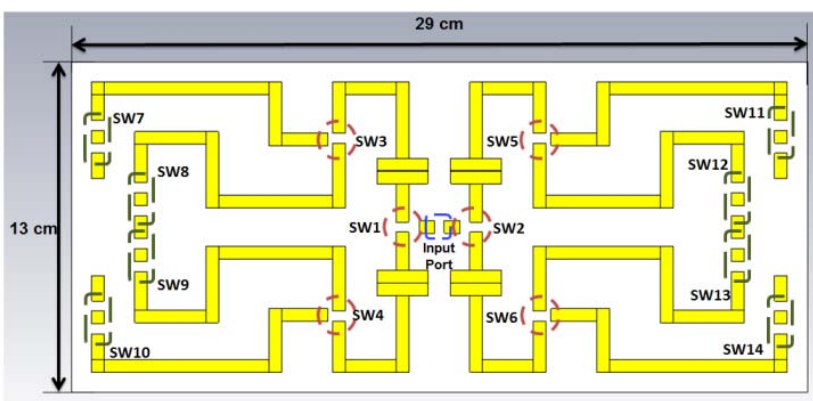

(a)

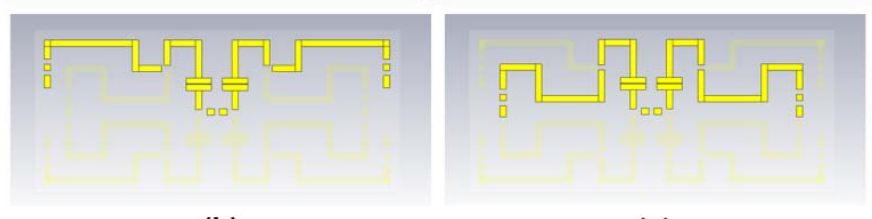

(b)

(c)

Fig. 1. (a) Antenna Geometry, (b) Principal Outer Branch, (c) Principal Inner Branch. 
The above antenna layout uses a large portion of the surface available on the off-the-shelf floating element (gray rectangle in Fig. 1a; size: $13 \times 29 \mathrm{~cm}$ ). The number of possible radiating configurations has been limited to 10 , in order to reduce the time required by an exhaustive search algorithm to determine the antenna shape with the best performance.

The two main identified configurations (Fig. 1b and Fig. 1c) have been optimized for free space operation (Fig. 2). Hereafter, they are denoted "Conf\#1" and "Conf\#2" for the outer and inner branch topology, respectively.

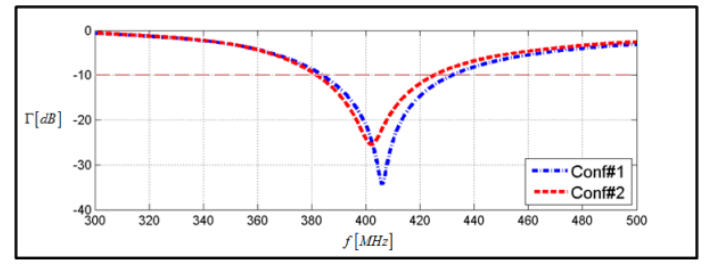

Fig. 2. Simulated $\mathrm{S}_{11}$ for "Conf\#1" and "Conf\#2" configurations shown in Fig. 1.

The latter configurations have been used as a reference for validating the performance of the self-structuring antenna during a number of floating antenna orientations with respect to the water level (see Fig. 4). Simulations have been conducted for different immersion distances $d$ of that part of the floating element immersed into water in a foundering situation and for different rotation angles, $\alpha, \beta$ when the floating element rotates around its orthogonal antenna axes (Fig. $4 \mathrm{~b}$ and Fig. 4c).

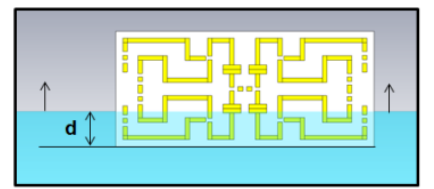

(a)

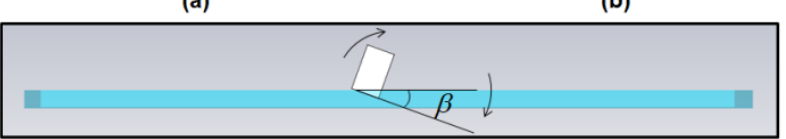

(c)

Fig. 4. (a) Antenna foundering, (b) Lateral Rotation, (c) Frontal Rotation (antenna is printed on the right side of the floating element).

For a set of values of $d, \alpha$ and $\beta$, numerical results in Fig. 5 show the minimum value for the reflection coefficient at $406 \mathrm{MHz}$, which has been obtained by a proper selection of all switches state. It is apparent that the antenna re-configurability improves the $S_{11}$ with respect to both nominal configurations Conf\#1/Conf\#2. A correct transmission condition is guaranteed $\left(\mathrm{S}_{11}\right.$ lower than $\left.-10 \mathrm{~dB}\right)$ even if the antenna sinks into the water by $6 \mathrm{~cm}$ (more than $45 \%$ of the antenna volume under the water), or when performing a lateral/frontal rotation up to $40^{\circ}$.

\section{CONCLUSION}

The layout of a reconfigurable multi-branch meanderedlike dipole has been proposed to implement a life-jacket integrated self-structuring antenna of a Cospas-Sarsat personal locator beacon. Numerical simulations have been performed with the antenna floating on the water. It has been shown that, through a proper switch configuration, it is possible to guarantee a return loss greater than $10 \mathrm{~dB}$ even in extreme floating conditions. Due to the presence of the water, the antenna radiation pattern looks like a relatively wide pattern pointing toward the zenith direction, regardless the switch combination. Future work will include the simulation of the designed antenna in presence of the human body, and the realization of an antenna prototype together with a microcontroller-based circuit for antenna self-reconfigurability.

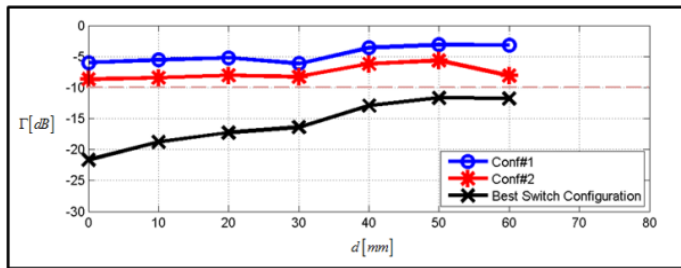

(a)

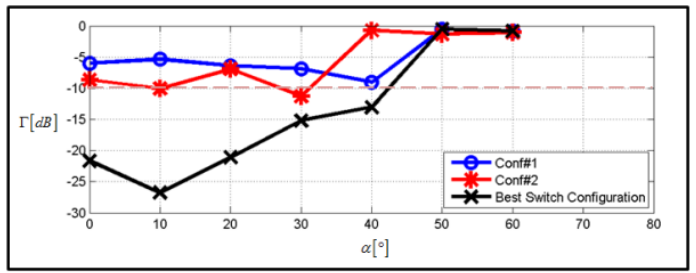

(b)

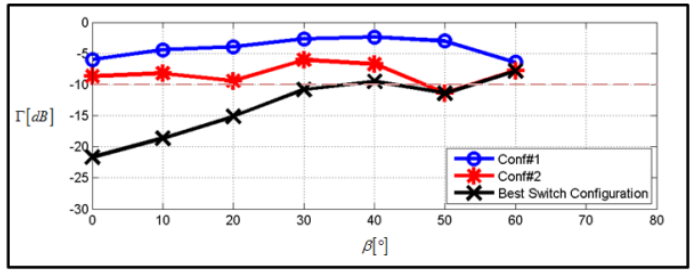

(c)

Fig. 5. Simulated $\mathrm{S}_{11}$ for different floating antenna positions with respect to water level: (a) antenna sinking (Fig. 4a); (b) lateral rotation (Fig. 4b); (c) frontal rotation (Fig. 4c).

\section{REFERENCES}

[1] A.A. Serra, P. Nepa, and G. Manara, "A Wearable Two-Antenna System on a Life Jacket for Cospas-Sarsat Personal Locator Beacons," IEEE TAP , vol.60(2), pp.1035-1042, Feb. 2012.

[2] J. Lilja, V. Pynttari, T. Kaija, R. Makinen, E. Halonen, H. Sillanpaa, J. Heikkinen, M. Mantysalo, P. Salonen and P. de Maagt, "Body-Worn Antennas Making a Splash: Lifejacket-Integrated Antennas for Global Search and Rescue Satellite System," IEEE Antennas and Propagation Magazine, vol. 55(2), pp. 324-340, April 2013.

[3] R.D. Seager, A. Chauraya, J.C. Vardaxoglou, P. de Maagt, "Towards a compact low frequency woven antenna," IEEE 2009 APS, pp. 1-4, 2009.

[4] C.M. Coleman, E.J. Rothwell and J.E. Ross, "Self-structuring antennas," IEEE 2000 APS , vol.3, pp.1256-1259, 16-21 July, 2000.

[5] C.M. Coleman, E.J. Rothwell, J.E. Ross, "Investigation of Simulated annealing, ant-colony optimization, and genetic algorithms for selfstructuring antennas," IEEE TAP, vol.52(4), pp.1007,1014, April 2004.

[6] B.T. Perry, C.M. Coleman, B.F. Basch, E.J. Rothwell, and J.E. Ross, J, "Self-structuring antenna for television reception," IEEE 2001 APS, pp.162-165, 8-13 July, 2001.

[7] B.T. Perry, E.J. Rothwell, L.L. Nagy, J.E. Ross, "Self-structuring antenna concept for FM-band automotive backlight antenna design," IEEE 2005 APS , pp.92-95, 2005. 